\title{
Stretchable Nanocomposite Sensors, Nanomembrane Interconnectors, and Wireless Electronics Toward Feedback-Loop Control of a Soft Earthworm Robot
}

Riccardo Goldoni ${ }^{\mathrm{a}, \mathrm{b}, \dagger}$, Yasemin Ozkan-Aydin ${ }^{\mathrm{c}, \dagger}$, Yun-Soung Kim ${ }^{\mathrm{a}, \dagger}$, Jongsu Kim ${ }^{\mathrm{a}}$, Nathan Zavanelli $^{\mathrm{a}, \mathrm{d}}$, Musa Mahmood ${ }^{\mathrm{a}}$, Bangyuan Liu ${ }^{\mathrm{a}}$, Frank L. Hammond III ${ }^{\mathrm{a}, \mathrm{e}}$, Daniel I. Goldman ${ }^{\mathrm{c}}$, and Woon-Hong Yeo ${ }^{\mathrm{a}, \mathrm{e}, \mathrm{f}, *}$

${ }^{a}$ George W. Woodruff School of Mechanical Engineering, Institute for Electronics and Nanotechn ology, Georgia Institute of Technology, Atlanta, GA 30332, USA.

${ }^{\mathrm{b}}$ School of Materials Science and Engineering, Georgia Institute of Technology, Atlanta, GA 30332, USA.

'School of Physics, Georgia Institute of Technology, Atlanta, GA 30332, USA.

${ }^{\mathrm{d} S c h o o l ~ o f ~ E l e c t r i c a l ~ a n d ~ C o m p u t e r ~ E n g i n e e r i n g, ~ G e o r g i a ~ I n s t i t u t e ~ o f ~ T e c h n o l o g y, ~ A t l a n t a, ~ A t l a n t ~}$ a, GA 30332, USA.

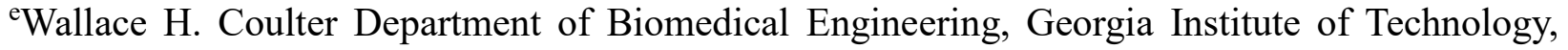
Atlanta, GA 30332, USA.

fParker H. Petit Institute for Bioengineering and Biosciences, Neural Engineering Center, Institute for Materials, Institute for Robotics and Intelligent Machines, Georgia Institute of Technology, Atlanta, GA 30332, USA.

$\dagger$ These authors contributed equally.

*Address correspondence to whyeo@gatech.edu 


\section{Note S1. Fabrication of nanocomposite sensors and electronics.}

Fabrication of the nanocomposite sensor

1. Cover a glass slide with PVA film fixing it to the back of the glass slide with tape.

2. Prepare the Ecoflex 00-30 formulation by mixing the equal parts of A and B in a plastic cup thoroughly for 1 minute.

3. Using a plastic pipette, draw $3 \mathrm{~g}$ of the formulation and dispense on the PVA film.

4. Facilitate homogeneous spreading of the silicone by tilting the substrate on different angles.

5. Let the elastomer cure at room $\mathrm{T}$ on a leveled surface for 4 hours.

6. Prepare the shadow mask based on the CAD design in Fig. S2 using a femtosecond laser cutter (Optec WS Flex).

7. Place the substrate on a hot plate at $80^{\circ} \mathrm{C}$ and lay the shadow mask.

8. Deposit MWCNT inside the opening of the shadow mask.

9. Spread the MWCNTs using a plastic squeegee. Repeat the procedure going from left to right of the pattern for 5 times.

10. Remove the excess MWCNTs powder on top of the shadow mask using a plastic squeegee.

11. Gently lift up the shadow mask from the elastomer substrate.

12. Let it rest for 5 minutes after screen printing.

13. Apply Ag stretchable ink (XE184E, Namics) to one of the leads of the screen-printed trace.

14. Using tweezers, attach one lead of a stretchable interconnector to the lead of the screenprinted trace, where the Ag stretchable ink has been applied.

15. Apply additional Ag stretchable ink to reinforce the connection.

16. Repeat the procedure for the other lead of the strain sensor.

17. Place the substrate on a hot place at $70^{\circ} \mathrm{C}$ and let the $\mathrm{Ag}$ stretchable ink cure for $30 \mathrm{~min}$.

18. Remove the substrate from the hot plate and let it rest for 2 minutes.

19. Prepare the Ecoflex 00-30/Slo-Jo formulation for the encapsulating layer. Pour the base component of the Ecoflex 00-30 formulation and 4\% in weight of Slo-Jo cure retardant (with respect to the Ecoflex 00-30 base component) in a plastic cup and stir thoroughly for $2 \mathrm{~min}$.

20. Add the Ecoflex 00-30 curing agent in a 1:1 ratio with the base component in the same plastic cup and stir thoroughly for $1 \mathrm{~min}$.

21. Using a plastic pipette, draw $3 \mathrm{~g}$ of the formulation and dispense on the substrate.

22. Facilitate homogeneous spreading of the elastomer by tilting the substrate on different angles.

23. Let it cure at room $\mathrm{T}$ on a flat surface for 8 hours.

24. Using a razor blade, cut out the area including the strain sensor from the substrate.

25. Remove the strain sensor from the glass slide by lifting the underlying PVA film from the glass. Dissolve PVA under a flowing water for 5 minutes.

Fabrication of stretchable interconnectors

1. Spincoat PDMS (4:1 base-curing-agent ratio) on a silicon wafer at $4000 \mathrm{RPM}$ for $30 \mathrm{sec}$, and then soft bake at $150{ }^{\circ} \mathrm{C}$ for $5 \mathrm{~min}$ on a hot plate.

2. Attach a Kapton film with 12.5 um over the wafer coated with PDMS. 
3. Cr deposition with $50 \AA$ thickness and $0.7 \AA /$ sec rate using Denton Explorer E-beam Evaporator.

4. Au deposition with $2000 \AA$ thickness and $3.0 \AA$ /sec rate using Denton Explorer E-beam Evaporator.

5. Spincoat photoresist (PR, Microposit SC1813, MicroChem) at 3000 RPM for $30 \mathrm{sec}$, and bake at $100{ }^{\circ} \mathrm{C}$ for $3 \mathrm{~min}$.

6. Align with a photomask and expose UV light with hard contact for $12 \mathrm{sec}$.

7. Develop UV-treated PR with a developer (MF-319, MicroChem).

8. Etch exposed Au with Au etchant (GE-8110, Transene).

9. Etch exposed $\mathrm{Cr}$ with $\mathrm{Cr}$ etchant (Chrome Mask Etchant 9030, Transene).

10. Remove remaining PR with acetone and foam swab, and rinse with IPA and DI water.

11. Spincoat 2nd PI layer (PI-2545, HD MicroSystems) at 4000 RPM for $60 \mathrm{sec}$, and cure on a hot plate at $100{ }^{\circ} \mathrm{C}$ for $5 \mathrm{~min}$ and at $300^{\circ} \mathrm{C}$ for $1 \mathrm{hr}$.

12. Spincoat PR (AZ P4620, Integrated Micro Materials) at 2000 RPM for $30 \mathrm{sec}$, and bake at $100^{\circ} \mathrm{C}$ for $4 \mathrm{~min}$.

13. Align with a photomask and expose UV light with hard contact for $120 \mathrm{sec}$.

14. Develop exposed PR with a developer (AZ-400K, Integrated Micro Materials) diluted with DI water (AZ400K:DI water $=1: 3$ ).

15. Etch exposed PI with reactive ion etcher (RIE) at $200 \mathrm{~W}, 150 \mathrm{mTorr}$, and $20 \mathrm{sccm}$ of oxygen for 45 min.

16. Remove remaining PR with acetone and foam swab, and rinse with IPA and DI water.

17. Repeat Step 12-16 one more time to completely etch PI.

18. Remove residues with acetone and foam swab, and rinse with IPA and DI water.

19. Peel off the microfabricated stretchable interconnector.

Integration of electronics components and encapsulation of the flexible circuit board

1. Transfer the flexible circuit board on a glass slide.

2. Screen-print low-temperature solder paste (alloy of $\mathrm{Sn} / \mathrm{Bi} / \mathrm{Ag}(42 \% / 57.6 \% / 0.4 \%)$, SMDLTLFP10T5, ChipQuik Inc.) with stainless-steel stencil on the top surface of circuitry.

3. Mount the electronics components.

4. Reflow solder the components on a hot plate by applying heat according to the temperature profile recommended by the solder paste manufacturer.

5. Prepare Ecoflex 00-30 mixture and cover the electronics without covering the sensor and power pads.

6. Integrate the battery and the switch to the flexible circuit board using Kapton tape. Fix it to the back of the board to guarantee stability.

7. Connect the non-encapsulated stretchable interconnectors leads coming from both the strain sensors to the 4 output pads of the flexible circuit board using silver paste (Fast Drying Silver Paint, Ted Pella).

Integration of strain sensor and electronic system on the body of the earthworm

1. Prepare the Solaris formulation by mixing the two parts in a plastic cup in a 1:1 ratio.

2. Stir thoroughly for $5 \mathrm{~min}$.

3. Apply the Solaris on the back of the strain sensor.

4. Place the strain sensor in the desired location on one side of the soft robot body. 
5. Using a hot air gun at $150^{\circ} \mathrm{C}$, selectively cure the borders and corners of the strain sensor to immobilize it on the body of the robot.

6. Let the remaining Solaris between the robot body and the back of the sensor cure at room T for 6 hours.

7. Encapsulate the stretchable interconnectors on the body of the robot by pouring Solaris and let cure for 6 hours.

After the curing process is over, use IPA to clean the body of the robot from unwanted material. 

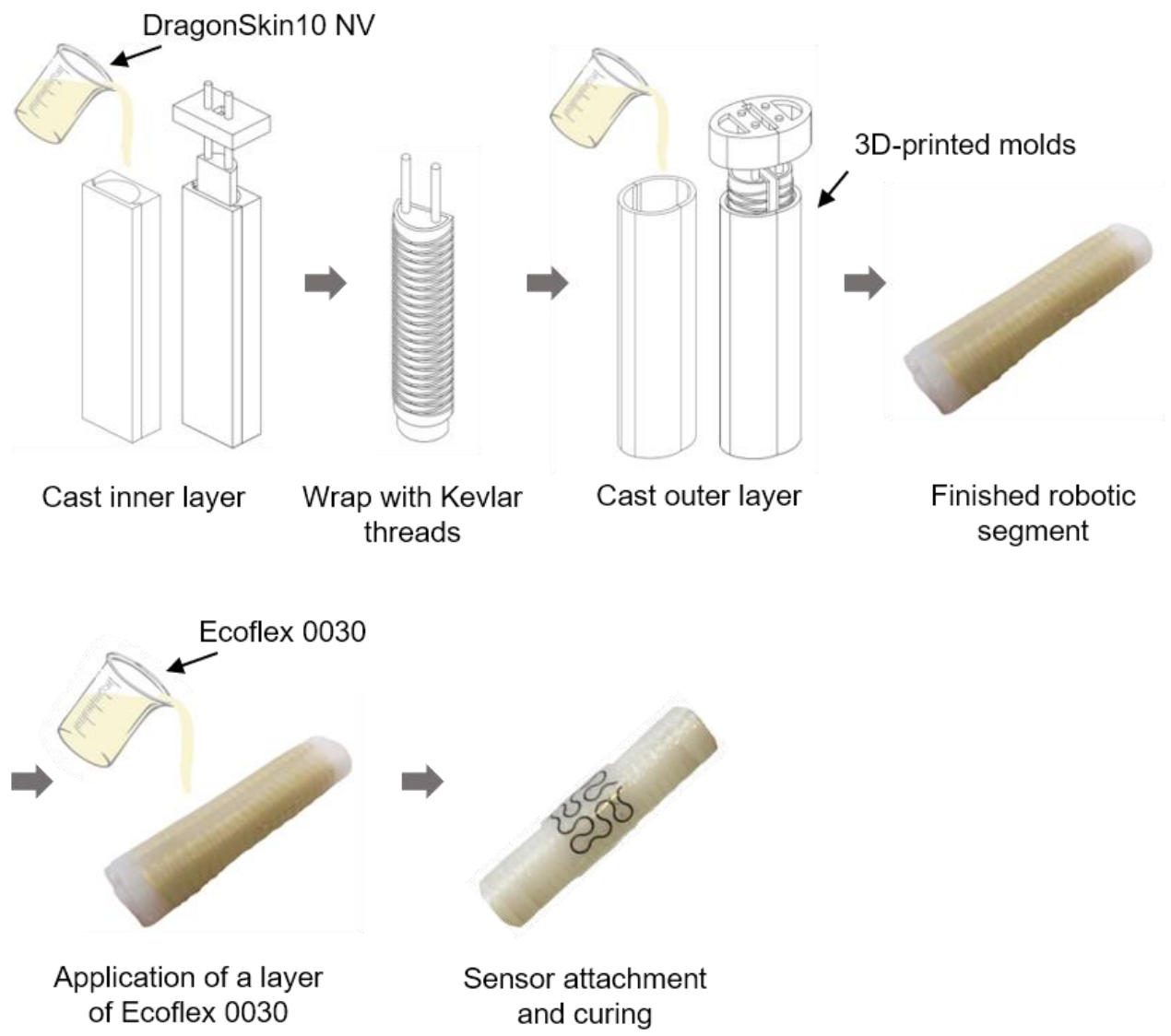

Figure S1. Fabrication process and structural description of a soft robotic segment. Schematics show elastomer casting, Kevlar threading steps for the fabrication of a soft earthworm segment, and sensor integration using Ecofelx 0030 as the glue layer. 


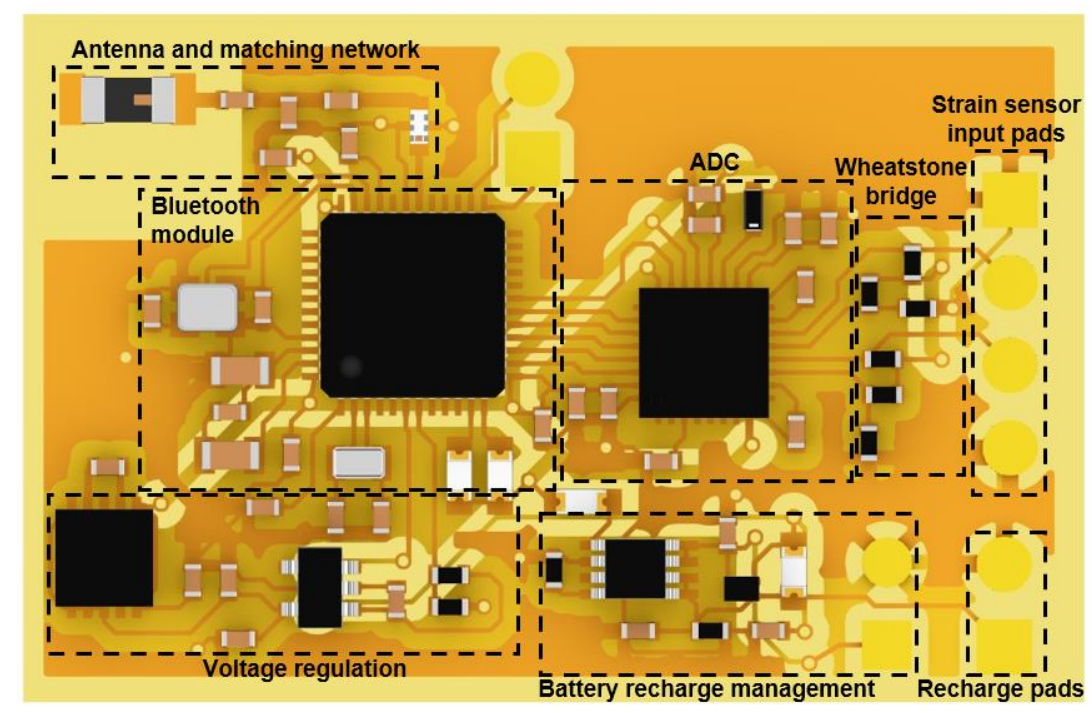

Figure S2. Design of a flexible circuit. Top view illustration of flexible circuitry and electronic components with functional block descriptions. 


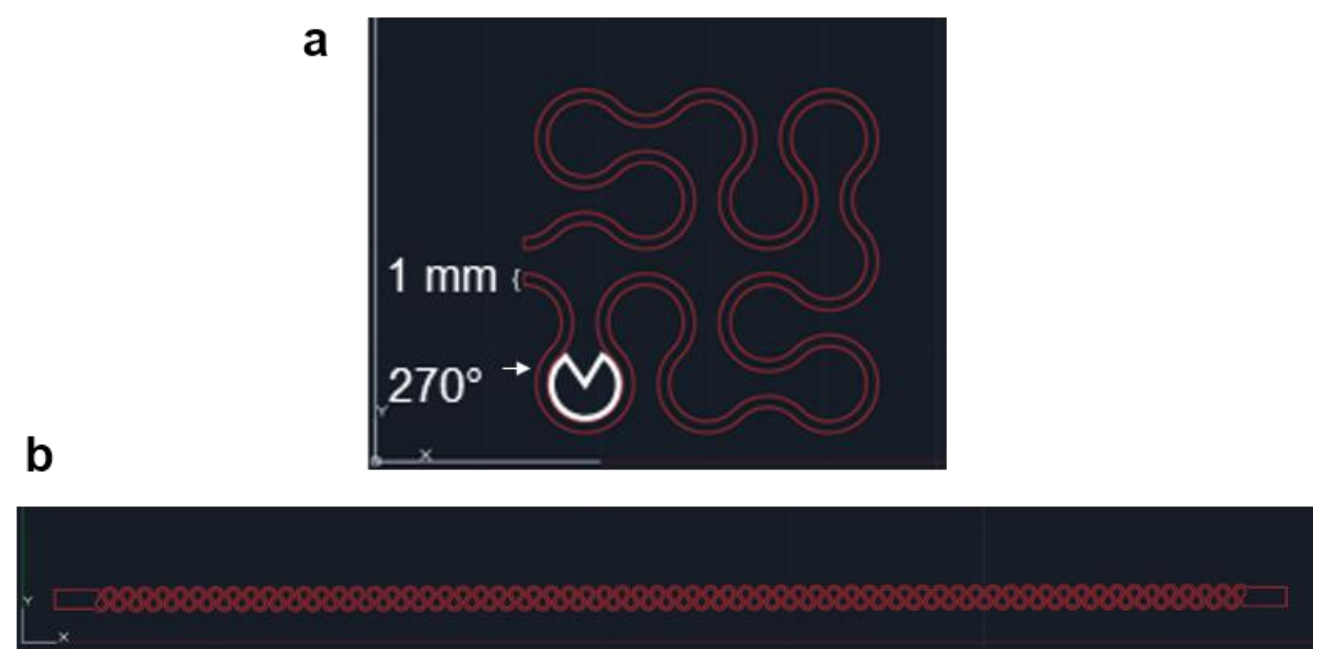

Figure S3. Designs for sensors and interconnectors. (a) AutoCAD design for the serpentine CNT trace. The parameters used in designing the serpentine trace are reported on the figure. Such design is then imported in the laser cutter software to realize the shadow mask for screen printing. (b) AutoCAD design for the stretchable interconnectors. 
a

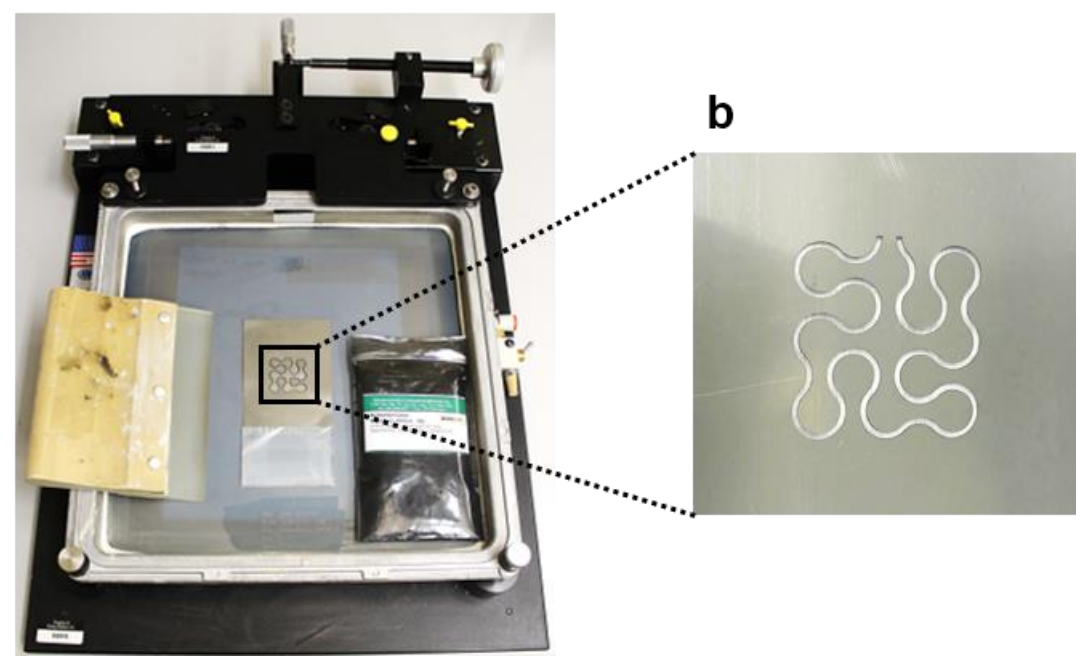

Figure S4. Experimental setup for screen printing. (a) Experimental setup for screen printing including the system and materials. (b) Close-up photograph of the laser cut stainless steel shadow mask used for CNT deposition. 


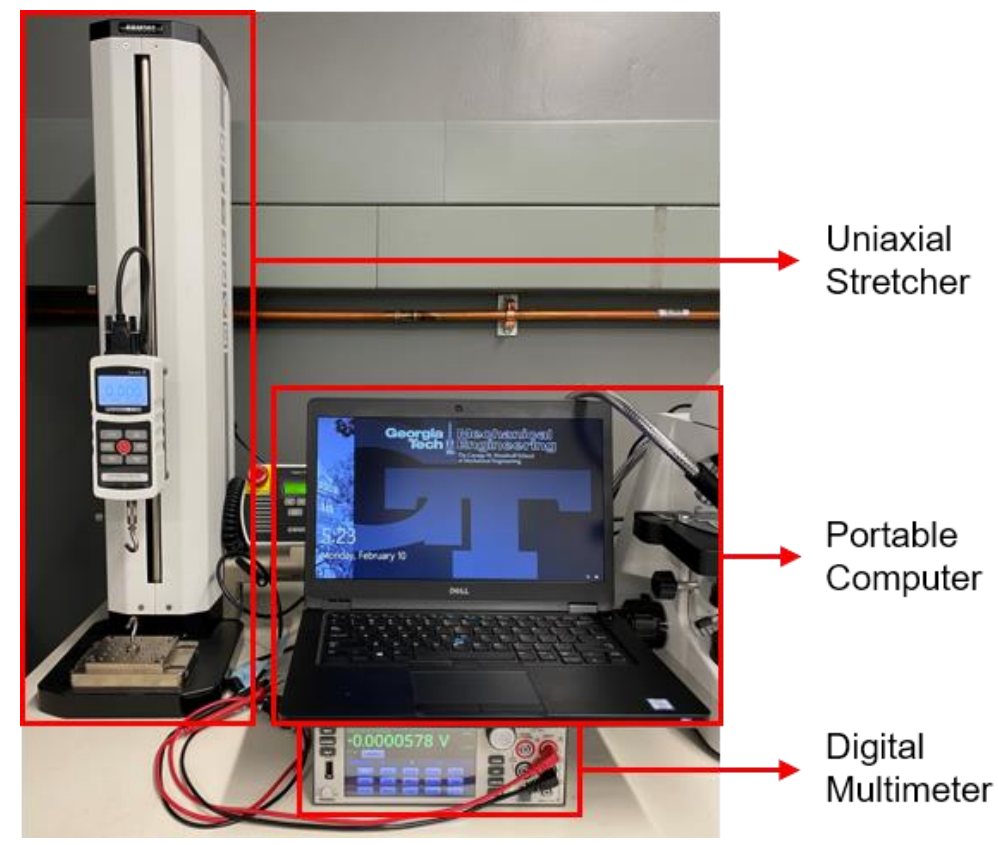

Figure S5. Experimental setup for uniaxial stretching. The laptop is connected via USB to both instruments and simultaneously records the displacement data over time from the uniaxial stretcher, while recording the changes in resistance from the digital multimeter. Flat clamps are used as multimeter leads instead of standard alligator clips due to the ultrathin nature of the stretchable interconnectors. 
Table S1. List of electronic components for a printed circuit board.

\begin{tabular}{|c|c|c|c|}
\hline Component & Description & Value & Part number \\
\hline U1 & 3.3 voltage regulator & N/A & TPS63001 \\
\hline $\mathrm{U} 2$ & Analog front-end & N/A & ADS1292 \\
\hline U3 & Bluetooth PSoC & N/A & NRF52832-QFAA-R \\
\hline U5 & Current limit active-low load switch & N/A & TPS22941 \\
\hline U6 & Charge management controller & N/A & MCP73831 \\
\hline L1 & 0402 inductor & $2.2 \mu \mathrm{H}$ & N/A \\
\hline L3 & 0402 inductor & $15 \mathrm{nH}$ & N/A \\
\hline L4 & 0603 inductor & $10 \mu \mathrm{H}$ & N/A \\
\hline L5 & 0402 inductor & $10 \mathrm{nH}$ & N/A \\
\hline L6 & 0402 inductor & $2.7 \mathrm{nH}$ & N/A \\
\hline $\mathrm{C} 1, \mathrm{C} 10, \mathrm{C} 14, \mathrm{C} 15$ & 0402 ceramic capacitor & $10 \mu \mathrm{F}$ & N/A \\
\hline $\mathrm{C} 2$ & 0402 ceramic capacitor & $22 \mu \mathrm{F}$ & N/A \\
\hline $\mathrm{C} 3, \mathrm{C} 5$ & 0402 ceramic capacitor & $4.7 \mathrm{nF}$ & N/A \\
\hline $\begin{array}{c}\text { C6, C7, C12, C22, } \\
\text { C26, C33 }\end{array}$ & 0402 ceramic capacitor & $0.1 \mu \mathrm{F}$ & N/A \\
\hline $\mathrm{C} 8, \mathrm{C} 11, \mathrm{C} 13$ & 0402 ceramic capacitor & $1.0 \mu \mathrm{F}$ & N/A \\
\hline C9 & 0402 tantalum capacitor & $1.0 \mu \mathrm{F}$ & N/A \\
\hline $\mathrm{C} 21$ & 0402 ceramic capacitor & $4.7 \mu \mathrm{F}$ & N/A \\
\hline $\mathrm{C} 23$ & 0603 ceramic capacitor & $10 \mu \mathrm{F}$ & N/A \\
\hline $\mathrm{C} 24, \mathrm{C} 25, \mathrm{C} 28, \mathrm{C} 29$ & 0402 ceramic capacitor & $12 \mathrm{pF}$ & N/A \\
\hline $\mathrm{C} 30$ & 0402 capacitor & $1.0 \mathrm{pF}$ & N/A \\
\hline $\begin{array}{l}\mathrm{R} 1, \mathrm{R} 2, \mathrm{R} 3, \mathrm{R} 4, \mathrm{R} 6, \\
\text { R11 }\end{array}$ & 0402 resistor & $200 \mathrm{k} \Omega$ & N/A \\
\hline $\mathrm{R} 5, \mathrm{R} 7$ & 0402 resistor & $1 \mathrm{M} \Omega$ & N/A \\
\hline R9 & 0402 resistor & $2 \mathrm{k} \Omega$ & N/A \\
\hline R10 & 0402 resistor & $100 \mathrm{k} \Omega$ & N/A \\
\hline A1 & 2.45 GHz RF chip antenna & N/A & 2450AT18A100 \\
\hline $\mathrm{F} 1$ & $2.45 \mathrm{GHz}$ low pass filter & N/A & 2450FM07A0029 \\
\hline $\mathrm{X} 1$ & $32 \mathrm{MHz}$ crystal & N/A & ECS-320-8-37CKM \\
\hline $\mathrm{X} 2$ & $32.768 \mathrm{kHz}$ crystal & N/A & ECS-.327-9-12-TR \\
\hline D1, D3 & Blue LED & N/A & LTST-C194TBKT \\
\hline D2 & Green LED & N/A & APT1608ZGCK \\
\hline D4 & Diode & N/A & CDBU0530 \\
\hline Q1 & MOSFET & N/A & DMP21D5UFB4-7B \\
\hline
\end{tabular}


Video S1. Evaluation of sensor system via a cyclic forward stretching test.

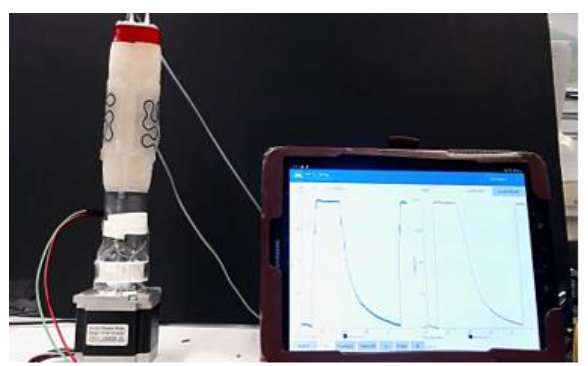

Forward stretching

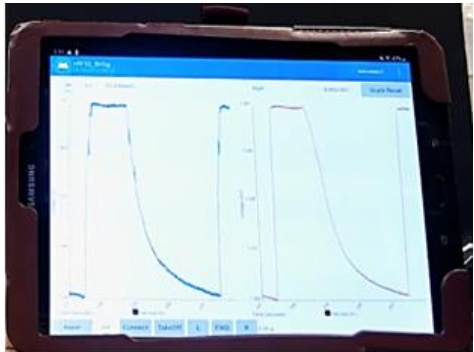

Tablet

Video S2. Evaluation of sensor system via a cyclic bending test.

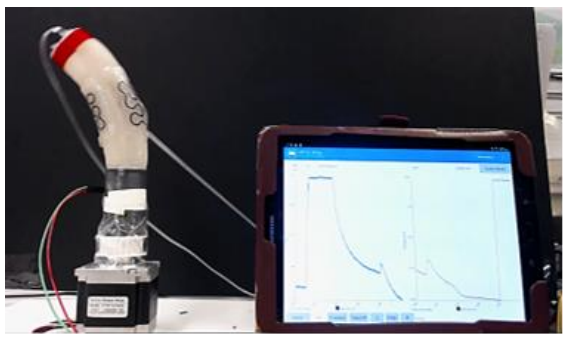

Left bending

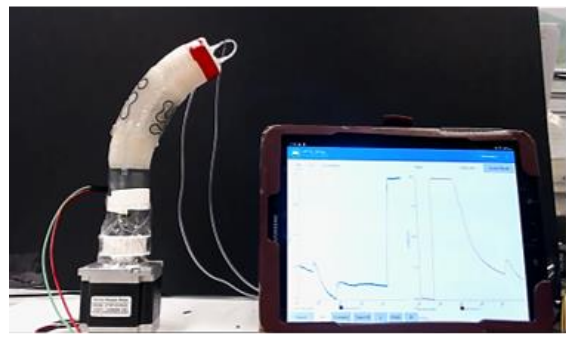

Right bending

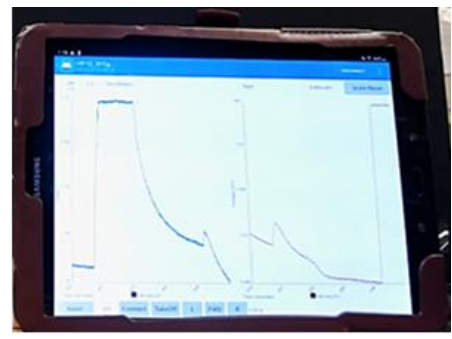

Tablet

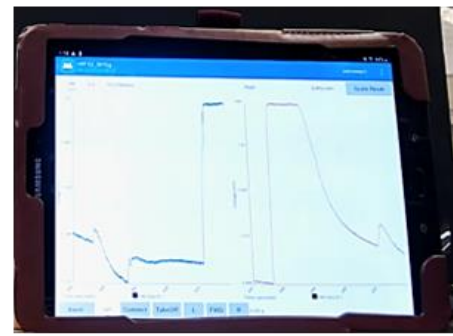

Tablet 